\title{
THE INDEPENDENCE OF CERTAIN AXIOMS OF STRUCTURES IN SETS ${ }^{1}$
}

\author{
JAPHETH HALL, JR.
}

\begin{abstract}
The independence of the axioms for spans and the independence of the axioms for closure structures are usually taken for granted. In this paper, the author establishes the independence of monotonicity, extensiveness, idempotence, the exchange property, the property of having $\varnothing$ as a fixed set and two covering properties ( $\alpha$-character, with $\alpha$ being some cardinal number, and a covering property with respect to generators). The independence of the axioms for closure structures and spans follow immediately. It is shown that any proof of the independence of a given axiom must involve an example with certain restrictions on the cardinal number $\alpha$.
\end{abstract}

Each of the following axioms or properties pertaining to structures in sets, that is, functions $P: 2^{V} \rightarrow 2^{V}$ for some set $V$, has been considered in at least one of [1]-[7]: monotonicity [if $X \subseteq Y \subseteq V$, then $P(X) \subseteq P(Y)$ ], extensiveness [if $X \subseteq V$, then $X \subseteq P(X)$ ], idempotence [if $X \subseteq V$, then $P(P(X))=P(X)$ ], the exchange property [if $X \subseteq V, y \in V, x \in P(X \cup\{y\})$ and $x \notin P(X)$, then $y \in P(X \cup\{x\})$ ], finite character [if $X \subseteq V$, then $P(X)$ is a subset of $\bigcup\{P(Z): Z \subseteq X, Z$ is finite $\}$ ]. Structures having monotonicity, extensiveness, and idempotence are called "closure structures" or "closure operations". (See, e.g., [1]-[6].) Closure structures having the exchange property and finite character are called "spans". (See, e.g., [2], [3], and [7].)

Finite character is a special case of the following property, where $\alpha$ is some cardinal number and $|S|$ denotes the cardinal number of a set $S$ :

$\alpha$-character. If $X \subseteq V$, then $P(X) \subseteq \bigcup\{P(Z): Z \subseteq X,|Z|<\alpha\}$. ( $\alpha$-character was considered in [4] and [5] for $\alpha$ not exceeding the first infinite cardinal number.) If $V$ is a vector space over a division ring and $P(X)$ is the subspace generated by $X$ for all $X \subseteq V$, then $P$ has the following property:

Equivalence covering property. If $X \subseteq V$ and $Y \subseteq V$ such that $P(X)=$ $P(Y)$, then $P(X) \subseteq \bigcup\{P(Z): Z \subseteq Y,|Z| \leqq|X|\}$.

Received by the editors October 12, 1970.

AMS 1970 subject classifications. Primary 06A20, 08-00; Secondary 15A03, 46A99, 50A99, 54-00.

Key words and phrases. Structures in sets, closure operations, finitary closure operations, spans.

${ }^{1}$ Work done while the author was writing a dissertation in partial fulfillment of requirements for the doctorate at the University of Alabama in 1969-1970.

(c) American Mathematical Society 1972 
It is usually assumed that the axioms for closure structures are independent, and that the axioms for spans are independent. It is the purpose of this paper to show the independence of monotonicity, extensiveness, idempotence, the exchange property, $\alpha$-character (with certain restrictions on $\alpha$ ), the equivalence covering property and the property of sending $\varnothing$ onto $\varnothing$. It will follow that any combination of these axioms is independent, and that the axioms for spans are independent.

Lemma. Suppose that $V$ is a set having a subset $A$ such that $V-A$ is nonempty. Let $F$ be a nonempty family of nonempty subsets of $V-A$. The following defines a structure $P$ in $V$ having monotonicity, idempotence, extensiveness and the property of sending $\varnothing$ onto $A$ :

$$
\begin{aligned}
& \text { If } X \subseteq V \text {, then } P(X)=V \quad \text { if } Y \subseteq X-A \text { for some } Y \in F \text {, } \\
& =X \cup A \text { otherwise, }
\end{aligned}
$$

where $S-T=\{x \in S: x \notin T\}$ for all sets $S$ and $T$. Moreover, if $\mu$ is a cardinal number such that $\mu \leqq|V|$, then the following conditions are satisfied:

(1) If $F$ is the family of all $Y \subseteq V$ such that $|Y|=\mu$, then $P$ has the exchange property.

(2) If $F$ is any family of subsets $Y$ of $V$ such that $|Y|=\mu$, then $P$ has the . equivalence covering property.

(3) If $\alpha$ is a cardinal number such that $\mu<\alpha$ and $F$ is any family of subsets $Y$ of $V$ such that $|Y|=\mu$, then $P$ has $\alpha$-character.

Proof. Since $X \subseteq X \cup A \subseteq V$ for each $X \subseteq V$, then $P$ has extensiveness. Assume that $X \subseteq Y \subseteq V$. Either $Z \subseteq X-A$ for some $Z \in F$ or not. If so, then $Z \subseteq Y-A$; hence, $P(X)=V=P(Y)$. If not, then $P(X)=X \cup A \subseteq Y \cup A \subseteq$ $P(Y)$. It follows that $P$ has monotonicity. Since $F \neq \varnothing$, then $Y \subseteq V-A$ for some $Y \in F$. Therefore, $P(V)=V$. It follows that $P(P(X))=P(X)$ if $P(X)=$ $V$. If $P(X)=X \cup A$, then $Y \nsubseteq X-A$ for all $Y \in F$; hence $Y \nsubseteq(X \cup A)-A$ for all $Y \in F$, and it follows that $P(X \cup A)=(X \cup A) \cup A=P(X)$. It follows that $P$ has idempotence. Since $Y \neq \varnothing$ for all $Y \in F$, then $Y \notin \varnothing-A$ for all $Y \in F$; hence, $P(\varnothing)=\varnothing \cup A=A$. This completes a proof of the first statement in the lemma. Assume that $\mu$ is a cardinal number such that $\mu \leqq|V|$. Suppose that $F$ is the family of all subsets $Y$ of $V$ such that $|Y|=\mu$ while $X \subseteq V, y \in V, x \in P(X \cup\{y\})$ and $x \notin P(X)$. It follows that $Y \subseteq[X \cup\{y\}]-A$ for some $Y \in F$ or not. We consider the case that $Y \subseteq[X \cup\{y\}]-A$ for some $Y \in F$. Since $P$ has extensiveness and $x \in P(X \cup\{y\})$ while $x \notin P(X)$, then $x \notin X$ and $y \notin X$. Therefore, if $Y \subseteq X$, then $y \in Y$. It follows that

$$
[Y-\{y\}] \cup\{x\} \subseteq[X \cup\{x\}]-A
$$

while, also $|[Y-\{y\}] \cup\{x\}|=\mu$; hence $P(X \cup\{x\})=V$ and, therefore, 
$y \in P(X \cup\{x\})$. In case for all $Y \in F, Y \nsubseteq[X \cup\{y\}]-A$, then $P(X \cup\{y\})-$ $P(X)=\{y\}$ and, therefore, $y=x$ so $y \in P(X \cup\{x\})$. This proves that (1) is satisfied. Assume that $F$ is any family of subsets $Y$ of $V$ such that $|Y|=\mu$ while $X$ and $Y$ are subsets of $V$ such that $P(X)=P(Y)$. It follows that $Z_{1} \subseteq X-A$ for some $Z_{1} \in F$ if and only if $Z_{2} \subseteq Y-A$ for some $Z_{2} \in F$; hence, $P(X)=V$ if and only if $P(Y)=V$. Therefore, $P$ has the equivalence covering property. This proves that (2) is satisfied. Assume that $\alpha$ is a cardinal number such that $\mu<\alpha$, and that $F$ is any family of subsets $Y$ of $V$ such that $|Y|=\mu$ while $X \subseteq V$. If $Y \subseteq X-A$ for some $Y \in F$, then $Y \subseteq X$ and $Y \subseteq Y-A$, so that $P(Y)=V$ while $|Y|=\mu<\alpha$. If for all $Y \in F, Y \subseteq X-A$ [so that $\mu \geqq 1]$ and $x \notin P(X)$, then $x \in(X \cup A)$; hence, either $x \in\{x\} \subseteq X$ while $x \in(\{x\} \cup A)=P(\{x\})$ or $x \in A=P(\varnothing)$. It follows that $P$ has $\alpha$-character. This proves that (3) is satisfied. The lemma follows.

THEOREM. The following axioms are independent: (It is to be understood that the restriction on $\alpha$ appearing alongside a given axiom is needed to prove the independence of that axiom.)

(a) The property of sending $\varnothing$ onto $\varnothing(\alpha>1)$.

(b) Monotonicity $(\alpha>2)$.

(c) Idempotence $(\alpha>2)$.

(d) Extensiveness $(\alpha \neq 2)$.

(e) The exchange property $(\alpha>1)$.

(f) $\alpha$-character $(\alpha>1)$.

(g) The equivalence covering property $(\alpha>\infty$, where $\infty$ denotes the first infinite cardinal number).

Proof. Excluding the case of the independence of idempotence, it is assumed that $V$ is a nonempty set. It is also assumed that $\mu$ is a cardinal number such that $2 \leqq \mu<|V|$. If $F$ is a nonempty family of nonempty subsets of $V-A$ for some $A \subseteq V$, then the symbol $P_{F, A}$ shall denote the structure $P$ defined in the lemma.

Independence of the property of sending $\varnothing$ onto $\varnothing$. Let $A$ be a nonempty subset of $V, \alpha$ be a cardinal number such that $\mu<\alpha$ and $F$ be the family of all $Y \subseteq V-A$ such that $|Y|=\mu$. It follows from the lemma that the independence of the property of sending $\varnothing$ onto $\varnothing$ is demonstrated with $P_{F, A}$.

Independence of monotonicity. Let $\alpha$ be a cardinal number such that $\mu<3 \leqq \alpha$. Let $F$ be the family of all $Y \subseteq V$ such that $|Y|=\mu$. Let $a, b$, and $c$ be distinct elements of $V$. Let $P=P_{F, \varnothing}$ and define a structure $Q$ in $V$ as follows:

$$
\text { If } \begin{aligned}
X \subseteq V \text {, then } Q(X) & =\{a, b\} & & \text { if } \varnothing \neq X \subseteq\{a, b\} \\
& =P(X) & & \text { otherwise. }
\end{aligned}
$$


Then $P(\{a\})=\{a, b\} \nsubseteq\{a, c\}=P(\{a, c\})$; hence, since $\{a\} \subseteq\{a, c\}$, it follows that $Q$ is not monotone. If $X$ is a nonempty subset of $\{a, b\}$, then it follows that $X \subseteq Q(X)=Q(Q(X))$ [since $Q(X)=\{a, b\}=Q(\{a, b\})$ ]. Since $P(\varnothing)=\varnothing$, then $\varnothing \subseteq Q(\varnothing)=Q(Q(\varnothing))$. If $X$ is a nonempty subset of $V$ such that $X \ddagger\{a, b\}$, then $Q(X)=P(X)$ while $P(X)$ is either $X$ or $V$; hence, $X \subseteq Q(X)=Q(Q(X))$. It follows that $Q$ is extensive and idempotent. Assume that $X \subseteq V, y \in V, x \notin Q(X)$, and $x \in Q(X \cup\{y\})$. If $X \cup\{y\}$ is a nonempty subset of $\{a, b\}$, then it follows that $Q(X \cup\{y\})-Q(X) \subseteq\{a, b\}$; hence, $x=a$ or $x=b$, and it follows that $y \in Q(X \cup\{x\})$ [since $y$ must also be either $a$ or $b$ and $X \cup\{x\}$ must be a nonempty subset of $\{a, b\}]$. We consider the case that $X \cup\{y\} \neq \varnothing$ and $X \cup\{y\}$ is not a subset of $\{a, b\}$. Then

$$
Q(X \cup\{y\})-Q(X)=P(X \cup\{y\})-Q(X) .
$$

Either $Q(X)=\{a, b\}$ or $Q(X)=P(X)$. In the latter case it follows that $y \in Q(X \cup\{x\})$ [since $P$ has the exchange property]. In the former case it follows that $x \notin\{a, b\}$ and, hence, $X \cup\{x\} \notin\{a, b\}$, so that $Q(X \cup\{x\})=$ $P(X \cup\{x\})$ while $P$ has the exchange property. Therefore, $y \in Q(X \cup\{x\})$ in either case. It follows that $Q$ has the exchange property. The sets which are $Q$-equivalent to $\{a, b\}$ are precisely $\{a\},\{b\}$, and $\{a, b\}$ while $P$ has the equivalence covering property. It follows that $Q$ has the equivalence covering property. Since $P$ has $\alpha$-character and $\alpha \geqq 3$, then $Q$ has $\alpha$ character. The independence of monotonicity follows.

Independence of idempotence. In this example we consider $V$ as a vector space over a division ring $F$ and assume that $V$ has dimension at least two. We let $a$ and $b$ be linearly independent elements of $V$ and $z=0$ be the identity in the Abelian group $V$. If $x \in V$ and $y \in V$, then we let $L(x, y)$ be the set of all $r x+x y$ such that $r \in F, s \in F$, and $r+s=1$. We let $Q(X)=\bigcup\{L(x, y): x \in X, y \in X\}$ for all $X \subseteq V$. Then $Q(Q(\{a, b, z\}))$ is the subspace spanned by $\{a, b\}$ while $Q(\{a, b, z\})$ is the proper subset $L(a, b) \cup L(a, z) \cup L(b, z)$ of $Q(Q(\{a, b, z\}))$. It follows that $Q$ is not idempotent. It is clear that $Q(\varnothing)=\varnothing$. Assume that $X \subseteq V$. It follows that $\{x\} \subseteq L(x, x) \subseteq Q(X)$ for all $x \in X$; hence, it follows that $Q$ is extensive. If $x \in X$ and $y \in X$ while $Y \subseteq V$ such that $X \subseteq Y$, then $x \in Y$ and $y \in Y$; hence, it follows that $Q(X) \subseteq Q(Y)$. Therefore, $Q$ is monotone. Assuming, further, that $y \in V, x \in Q(X \cup\{y\})$ and $x \notin Q(X)$, choose elements $c$ and $d$ of $X \cup\{y\}$ such that $x \in L(c, d)$. Since $x \notin Q(X)$, then one of $c$ and $d$ must be $y$, say $d=y$. Choose $r \in F$ and $s \in F$ such that $r+s=1$ and $x=r c+s y$. It is necessary that $s \neq 0$. Solving for $y$, one obtains the equation $y=s^{-1} x+\left(-s^{-1} r\right) c$ with $s^{-1}+\left(-s^{-1} r\right)=1$. It follows that $y \in Q(X \cup\{x\})$. Therefore, $Q$ has the exchange property. If $X$ is a nonempty subset of $V$ and $x \in Q(X)$, then one chooses $y \in X$ and $w \in X$ such that $x \in L(y, w)=Q(\{y, w\})$ while $|\{y, w\}|<\alpha$ if $\alpha$ is a cardinal number at least three. It follows that $Q$ has $\alpha$-character if 
$\alpha$ is a cardinal number at least three. Finally, if $X$ and $Y$ are subsets of $V$ such that $P(X)=P(Y)$ and $x \in Q(X)$, then $x \in L(y, w)=Q(\{y, w\})$ for some $\{y, w\} \subseteq Y$ while $|\{y, w\}| \leqq|X|$. It follows that $Q$ has the equivalence covering property. The independence of idempotence follows.

Independence of extensiveness. Let $\alpha$ be a cardinal number such that $\mu<\alpha$ and $F$ be the family of all $Y \subseteq V$ such that $|Y|=\mu$. Let $Q(X)=\varnothing$ if $Y \nsubseteq X \subseteq V$ for some $Y \in F$, and let $Q(X)=V$ if $Y \subseteq X \subseteq V$ for some $Y \in F$. Let $x \in V$. Then $\{x\} \nsubseteq Q(\{x\})$ if $\mu \geqq 2$, so that $Q$ is not extensive. It is easy to verify that $Q$ has all other properties listed in the statement of the theorem. Observe that the constant structure $Q$ such that $Q(X)=\varnothing$ for all $X \subseteq V$ suffices also.

Independence of the exchange property. Let $B$ be a nonempty subset of $V$ such that $|B|<\alpha$ (with $\alpha$ being a cardinal number) and $F=\{B\}$. Since $B \neq \varnothing$, we let $b \in B$. For $\alpha \leqq|V|$, we let $a \in(V-B)$. It follows that $a \in[V-(B-\{b\})], V-(B-\{b\})=P([B-\{b\}] \cup\{b\})$ and $b \notin[(B-\{b\}) \cup\{a\}]=$ $P([B-\{b\}] \cup\{a\})$. It follows that $P_{F, \varnothing}$ does not have the exchange property. It is clear that $P_{F, \varnothing}$ has the remaining properties listed in the statement of the theorem. The independence of the exchange property follows.

Independence of $\alpha$-character. Let $\alpha$ be a cardinal number less than $|V|$ and $F$ be the family of all $Y \subseteq V$ such that $|Y|=\alpha$. If $P_{F, \varnothing}$ has $\alpha$-character, then

$$
P_{F, \varnothing}(X) \subseteq \bigcup\left\{P_{F, \varnothing}(Y): Y \subseteq X,|Y|<\alpha\right\}=\bigcup\{Y: Y \subseteq X,|Y|<\alpha\}=X
$$

for all $X \subseteq V$. But $P_{F, \varnothing}(X)=V$ for some $X \in F$; hence, $V \subseteq X$ for some $X \in F$, so that $|V|=\alpha<|V|$. It follows that $P_{F, \varnothing}$ does not have $\alpha$-character. It is clear that $P_{F, \varnothing}$ has the remaining properties listed in the statement of the theorem. The independence of $\alpha$-character follows.

Independence of the equivalence covering property. Assume that $\alpha$ is a cardinal number such that $V$ includes two infinite subsets $A_{1}$ and $A_{2}$ such that $\left|A_{1}\right|<\left|A_{2}\right|<\alpha$. Let $F$ be the family of all $X \subseteq V$ such that $A_{i}-X$ is finite and $\left|X-A_{i}\right| \geqq\left|A_{i}-X\right|$ for some $i \in\{1,2\}$. Let $P=P_{F, \varnothing}$. Since $P\left(A_{1}\right)=P\left(A_{2}\right)$ and $x \notin P(Z)$ for all $Z \subseteq A_{2}$ such that $|Z| \leqq\left|A_{1}\right|$ and all $x \in V$ such that $x \in\left(V-A_{1}\right)$ while $V-A_{1} \neq \varnothing$, then $P$ does not have the equivalence covering property. Assume that $X \subseteq V, y \in V, x \in P(X \cup\{y\})$ and $x \notin P(X)$. It is clear that $y \in P(X \cup\{x\})$ if $X \cup\{Y\}=P(X \cup\{y\})$. Consider the case that $X \cup\{y\} \neq P(X \cup\{y\})$. Then $P(X \cup\{y\})=V$; hence, we choose $Y \in F$ such that $Y \subseteq[X \cup\{y\}]-\varnothing$. It follows that $A_{k}-Y$ is finite and $\left|Y-A_{k}\right| \geqq\left|A_{k}-Y\right|$ for some $k \in\{1,2\}$ and, therefore, $A_{k}-(Y \cup\{x\})$ is finite and

$$
\left|(Y \cup\{x\})-A_{k}\right| \geqq\left|A_{k}-(Y \cup\{x\})\right|
$$

while, also, $Y \cup\{x\} \subseteq(X \cup\{x\})-\varnothing$. Therefore, $Z \subseteq(X \cup\{x\})-\varnothing$ for some 
$Z \in F$ and, hence, $P(X \cup\{x\})=V$. It follows that $y \in P(X \cup\{x\})$. Therefore, $P$ has the exchange property. Excluding the exchange property and the equivalence property, it is clear that $P$ has the remaining properties listed in the statement of the theorem. The independence of the equivalence property follows. It remains to be shown that the restrictions on $\alpha$ are needed.

Assuming that $P$ is a structure in a set $V$, then by definition, a subset $X$ of $V$ is $P$-independent if and only if $x \notin P(X-\{x\})$ for all $x \in X$; a subset $X$ of $V$ is $P$-equivalent to a subset $Y$ of $V$ if and only if $P(X)=P(Y)$.

The following propositions regarding structures $P$ in sets $V$ are known. (See, e.g., [3] and [4] in which other references are also cited.)

(I) If $P$ is a span, then any two $P$-independent $P$-equivalent subsets of $V$ have the same cardinal number.

(II) If $P$ is a closure structure having the exchange property, then any two $P$-independent $P$-equivalent subsets of $V$, one of which is finite, have the same cardinal number.

(III) If $P$ has monotonicity and finite character, then every subset of $V$ has a maximal $P$-independent subset.

(IV) If $P$ has the exchange property and $X$ is a $P$-independent subset of $V$, then the following condition is satisfied:

$$
x \in[V-P(X)] \text { implies } X \cup\{x\} \text { is } P \text {-independent. }
$$

The following proposition will be proved:

(V) If $P$ is a structure in a set $V$ such that (i) and (ii) below are true, then $P$ has the equivalence covering property.

(i) Every subset $X$ of $V$ has a $P$-independent subset which is $P$ equivalent to $X$.

(ii) Any two $P$-independent $P$-equivalent subsets of $V$ have the same cardinal number.

Assume that $P$ is a structure in a set $V$ such that (i) and (ii) are true while $P$ does not have the equivalence covering property. Choose $P$ equivalent subsets $X$ and $Y$ of $V$ such that there is an $x \in P(X)$ such that $x \notin P(Z)$ for all $Z \subseteq Y$ such that $|Z| \leqq|X|$. Using (i), choose $P$-independent subsets $Z_{1}$ of $X$ and $Z_{2}$ of $Y$ such that $Z_{1}$ is $P$-equivalent to $X$ and $Z_{2}$ is $P$-equivalent to $Y$. Then $\left|Z_{2}\right|>|X| \geqq\left|Z_{1}\right|$. This contradicts (ii). It follows that $P$ has the equivalence covering property.

We will use (I) through (V) to explain certain restrictions in the case of the equivalence covering property. Assume that $P$ is a closure structure in a set $V$, that $P$ has $\alpha$-character and the exchange property, and that $P$ does not have the equivalence covering property. Since $P$ is a closure structure having the exchange property, it follows from (IV) that if $X \subseteq Y \subseteq V$, then $X$ is $P$-independent and $P$-equivalent to $Y$ if and only if 
$X$ is a maximal $P$-independent subset of $Y$. Therefore, it follows from (I), (III), and (V) that $\alpha>\infty$. This shows that the restriction on $\alpha$ in the case of the equivalence covering property is needed. It follows from (II) that the two infinite independent and equivalent sets used in the proof of the independence of the equivalence covering property are needed.

To explain the restrictions on $\alpha$ in the remaining cases, it suffices to consider the constant structure $Q$ having $\varnothing$ as its only value in a nonempty set $V$ along with a structure $P$ in $V$ defined as follows:

$$
\text { If } X \subseteq V \text {, then } P(X)=\bigcup\{P(Z): Z \subseteq X,|Z|<2\} \text {. }
$$

It is obvious that $Q$ is the only structure sending $\varnothing$ onto $\varnothing$ and having 1-character. Moreover, $Q$ has monotonicity, idempotence, the exchange property, and the equivalence covering property while $Q$ does not have extensiveness. Furthermore, the following is true:

(VI) $P$ has monotonicity, 2-character, and the equivalence covering property.

Also, the following conditions are satisfied:

(VII) $P$ has extensiveness if and only if $x \in P(\{x\})$ for all $x \in V$.

(VIII) If $P(\varnothing)=\varnothing$, then $P$ has the exchange property if and only if the following condition is satisfied by all $y \in V$ :

$$
P(\{y\}) \neq \varnothing \text { implies } y \in \bigcap\{P(\{x\}): x \in P(\{y\})\} .
$$

(IX) $P$ is idempotent if and only if $P(P(\{x\}))=P(\{x\})$ for all $x \in V$.

(X) If $X \subseteq V$, then $P(X)=P(\varnothing) \cup[\cup\{P(\{x\}): x \in X\}]$.

The truth of (VI) follows immediately from the definition of $P$. It is obvious that (VII) is satisfied, and that $(\mathrm{X})$ is satisfied. Suppose that $P(\varnothing)=\varnothing$. Assuming that $P$ has the exchange property while $y \in V$ and $x \in P(\{y\})$, then it follows that $x \in P(\varnothing \cup\{y\})$ while $x \notin P(\varnothing)$; hence, $y \in P(\varnothing \cup\{x\})$. Therefore, the condition in (VIII) is satisfied by all $y \in V$. Conversely, assuming that the condition in (VIII) is satisfied by all $y \in V$ while $X \subseteq V, y \in V, x \in P(X \cup\{y\})$ and $x \notin P(X)$, choose $z \in(X \cup\{y\})$ such that $x \in P(\{z\})$. It follows from the monotonicity of $P$ that $y=z$; hence, $x \in P(\{y\})$. Apply the condition in (VIII) to deduce that $y \in \bigcap\{P(\{z\}): z \in P(\{y\})\}$; hence, since $x \in P(\{y\})$ and $P$ has monotonicity, it follows that $y \in P(\{x\}) \subseteq$ $P(X \cup\{x\})$. Therefore, $P$ has the exchange property. This completes a proof of (VIII). Assume that $P(P(\{x\}))=P(\{x\})$ for all $x \in V$ while $X \subseteq V$ and $x \in P(P(X))$. Choose $y \in P(X)$ such that $x \in P(\{y\})$; then choose $z \in X$ such that $y \in P(\{z\}))$. Since $P$ has monotonicity and idempotence, it follows that $P(\{y\}) \subseteq P(P(\{z\}))=P(\{z\}) \subseteq P(X)$. Therefore, $x \in P(X)$. It follows that $P(P(X)) \subseteq P(X)$. Since $P$ has monotonicity, it follows that $P(X) \subseteq P(P(X))$. Therefore, it follows that $P$ has idempotence. It is obvious that if $P$ has 
idempotence, then $P(P(\{x\}))=P(\{x\})$ for all $x \in V$. This completes a proof of (IX).

We begin a discussion of the remaining restrictions on $\alpha$ in the statement of the theorem by assuming that $P(\varnothing)=\varnothing$ while $P$ has the exchange property and idempotence. If $P(\{y\}) \neq \varnothing$ for all $y \in V$, then it follows from (VIII) and the monotonicity of $P$ that $y \in \bigcap\{P(\{x\}): x \in P(\{y\})\} \subseteq P(P(\{y\}))=$ $P(\{y\})$ for all $y \in V$; hence, it follows from (VII) that $P$ is extensive. Since $P(\varnothing)=\varnothing$, then it follows that $\{x\}$ is $P$-independent for all $x \in V$. Consider the case that $P(\{y\})=\varnothing$ for some $y \in V$. Suppose that $P(\{z\}) \neq \varnothing$ for some $z \in V$. It follows from (VIII) that $y \notin P(\{z\})$. Since $\{x\}$ is $P$-independent for all $x \in V$, it follows from (IV) that $\{y, z\}$ is $P$-independent. Since $P$ has monotonicity, it follows that $P(\{z\}) \subseteq P(\{y, z\})=P(\{y\}) \cup P(\{z\})=P(\{z\})$; hence, it follows that $\{y, z\}$ is not $P$-independent. Therefore, the supposition that $P(\{z\}) \neq \varnothing$ for some $z \in V$ is false. It follows that $P(\{z\})=\varnothing$ for all $z \in V$; hence, it follows that $P(X)=\varnothing$ for all $X \subseteq V$. Therefore, $P=Q$. But $Q$ does not have 2-character while $P$ has 2 -character. Consequently, the case that $P(\{y\})=\varnothing$ for some $y \in V$ is not possible. This shows that there is a structure having $\alpha$-character and which can be used to demonstrate the independence of extensiveness if and only if $\alpha \neq 2$.

We now explain the restriction on $\alpha$ in the case of idempotence. Assume that $P(\varnothing)=\varnothing$ while $P$ is extensive and has the exchange property. Then $P(\{x\}) \subseteq P(P(\{x\}))$ for all $x \in V$. Either $P(P(\{x\})) \neq P(\{x\})$ for some $x \in V$ or not. Consider the case that $P(P(\{x\})) \neq P(\{x\})$ for some $x \in V$. If $P(\{y\})=\varnothing$ for all $y \in P(P(\{x\}))$ such that $y \notin P(\{x\})$, then $P([P(P(\{x\}))-P(\{x\})])=\varnothing$; hence, it follows from the extensiveness of $P$ that $P(P(\{x\}))=P(\{x\})$. It follows that $P(\{y\}) \neq \varnothing$ for some $y \in P(P(\{x\}))$ such that $y \notin P(\{x\})$. For such an element $y$ of $P(P(\{x\}))$, it follows that $y \in \bigcap\{P(\{z\}): z \in P(\{y\})\}$ while $y \in P(\{y\})$ [since $P$ is extensive] and $y \in[P(P(\{x\}))-P(\{x\})]$; hence, it follows from the monotonicity of $P$ that $y \in P([P(P(\{x\}))-P(\{x\})])$. Since $P(\varnothing)=\varnothing$, it follows from (X) that $P([P(P(\{x\}))-P(\{x\})])=P(P(P(\{x\})))-P(P(\{x\}))$; hence, $y \notin P(P(\{x\}))$. This latter result is contrary. It follows that there is no element $y$ of $P(P(\{x\}))$ such that $y \notin P(\{x\})$. Therefore, it follows that $P(P(\{x\})) \subseteq P(\{x\})$ and, hence, $P(P(\{x\}))=P(\{x\})$. Then, it follows from (IX) that $P$ is idempotent. It has already been observed that no structure having 1-character can be used to demonstrate the independence of idempotence. Therefore, the restriction $\alpha>2$ is needed to prove the independence of idempotence.

It has already been observed that the restriction $\alpha>1$ is needed to prove the independence of the property of sending $\varnothing$ onto $\varnothing$, the independence of the exchange property and the independence of monotonicity. It follows from (VI) that the restriction $\alpha>2$ is needed to prove the independence of monotonicity. The theorem follows. 


\section{BIBLIOGRAPHY}

1. G. Birkhoff, Lattice theory, 3rd ed., Amer. Math. Soc. Colloq. Publ., vol. 25, Amer. Math. Soc., Providence, R.I., 1967. MR 37 \#2638.

2. M. N. Bleicher and $\mathrm{E}$. Marczewski, Remarks on dependence relations and closure operators, Colloq. Math. 9 (1962), 209-212. MR 26 \#58.

3. M. N. Bleicher and G. B. Preston, Abstract linear dependence relations, Publ. Math. Debrecen 8 (1961), 55-63. MR 24 \#A124.

4. P. C. Hammer, Extended topology: domain finite expansive functions, Nieuw Arch. Wisk. (3) 9 (1961), 25-33. MR 26 \#6924.

5. - Extended topology: reduction of limit functions, Nieuw Arch. Wisk. (3) 9 (1961), 16-24. MR 26 \#6923.

6. R. S. Pierce, Introduction to the theory of abstract algebras, Holt, Rinehart and Winston, New York, 1968, pp. 15-30. MR 37 \#2655.

7. O. Zariski and P. Samuel, Commutative algebra. Vol. 1, University Series in Higher Math., Van Nostrand, Princeton, N.J., 1958. MR 19, 833.

Department of Mathematics, Stillman College, Tuscaloosa, Alabama 35401 